\title{
Epidemiological Studies Based on Small Sample Sizes - A Statistician's Point of View
}

\author{
By Annette Kjaer Ersbøll ${ }^{1}$ and Bjarne Kjar Ersbøll ${ }^{2}$
}

${ }^{1}$ Department of Animal Science and Animal Health, The Royal Veterinary and Agricultural University, Denmark, and ${ }^{2}$ Informatics and Mathematical Modelling, The Technical University of Denmark, Denmark

\begin{abstract}
Ersbøll AK, Ersbøll BK: Epidemiological studies based on small sample sizes - a statisticians point of view. Acta vet. scand. 2003. Suppl. 98, 127-140. - We consider 3 basic steps in a study, which have relevance for the statistical analysis. They are: study design, data quality, and statistical analysis. While statistical analysis is often considered an important issue in the literature and the choice of statistical method receives much attention, less emphasis seems to be put on study design and necessary sample sizes. Finally, a very important step, namely assessment and validation of the quality of the data collected seems to be completely overlooked.

Examples from veterinary epidemiological research and recommendations for each step are given together with relevant references to the literature.
\end{abstract}

small data set; design; data management; data quality; statistical analysis; sample size; power; simulation.

\section{Introduction}

There are 3 main issues in a study: design, data quality (data management), and statistical analysis (Fig. 1). In the statistical literature much emphasis is put on performing the correct statistical analysis. In contrast to this, standard statistical methods are often used in the veterinary literature and much more emphasis is put on interpreting the outcome of the statistical analysis. However, in neither case is the statistical analysis able to correct the consequences of a badly designed study or deal meaningfully with data of dubious quality. The aspects of choosing a sensible study design and the influence of sample size are usually not reported and are not

$$
\text { Design } \longrightarrow \text { Data management } \longrightarrow \text { Analysis }
$$

Figure 1. Three important phases in a study. very well represented outside purely statistical literature. In fact, in the authors' opinion surprisingly many statistical analyses are performed on data where little or no reflection at all has been made on study design and necessary sample size. Very often, just a few extra thoughts about a study design could have drastically improved the outcome of the study - in many cases at no extra cost or at a marginal extra cost. Furthermore, power calculations in the design phase can help design the study efficiently and stop studies that only have a small chance of supporting the study hypothesis. Finally, a nearly totally overlooked area is that of data quality. Even a carefully planned experiment can be rendered worthless if data collection is not performed correctly. After data collection data can be checked for errors in different ways, in some cases data are expected 
to behave according to some pattern, dates are known to be ordered for instance, growth curves are (usually) expected to grow monotonously, etc. Even more importantly, data can be monitored for consistency during collection, thereby permitting changes to the collection procedure to be made so possible misunderstandings are corrected at the source. For illustrative purposes examples of small data sets are given initially. We continue by describing experimental design, data quality and control and finally analysis of small data sets. The paper is concluded with perspectives and recommendations.

\section{Examples}

Four examples of small data sets are used for illustration:

\section{Example 1: Healing of wounds in horses}

The aim of this hypothetical study was to evaluate 2 different antibiotics (A and B) in combination with 2 bandages (I and II) for healing of wounds in horses. Selection of study design is the main problem in this example.

\section{Example 2: Moderate coliform mastitis}

The aim of the study was to evaluate avoidance of antibiotics in treatment of moderate coliform mastitis in dairy cows (Katholm \& Andersen 2001). Thirteen cows were included in the study $\left(\mathrm{CFU} / \mathrm{cm}^{2}<142\right), 7$ of these were treated with antibiotics. On day 21 the cows were evaluated for clinical recovery (restored or not).

\section{Example 3: Nematodes in sows and piglets}

The aim of the study was to investigate the influence of multiple nematode infections on piglet's performance (Thamsborg et al. 2001). Thirty-nine sows were included in the study and given one of 3 infection levels: control (12 sows), low triggered (13 sows) and high triggered (14 sows). Each litter was divided into 2 groups, one group in each litter was given an infectious dose, the other group in each litter was not. This example was included for its interesting design, even it is not a 'small data set'.

\section{Example 4: Milk yield in dairy cows}

How many herds and how many cows per herd should be included in the study in order to demonstrate a significant difference in milk yield for Jersey and Holstein cows? For illustration both the continuous outcome and a dichotomised outcome (milk yield above or below $18 \mathrm{~kg}$ ) have been used.

\section{Study design}

Design covers all the problems and considerations made before data collection starts. In the following aspects on design, sample size and power, compliance and randomisation will be discussed.

\section{Design}

Research studies are performed using either an observational design or an experimental design (Fig. 2). Most epidemiological research is based on observational studies. Observational studies are characterised by the fact that information of subjects is collected without affecting them in a pre-planned manner, often with little or no control on subjects. By contrast, in

\begin{tabular}{|l} 
Observational $\left\{\begin{array}{l}\text { Case-control } \\
\text { Cohort } \\
\text { Cross-sectional }\end{array}\right.$ \\
Experimental $\left\{\begin{array}{l}\text { Cross-over } \\
\text { Factorial (Parallel) } \\
\text { Latin square }\end{array}\right.$ \\
\hline
\end{tabular}

Figure 2. Examples of designs for observational and experimental studies. 


\begin{tabular}{llll}
\hline Design & Past & Present & Future \\
\hline Cohort & & Exposure $\longrightarrow$ Disease \\
Case-control & Exposure & Disease \\
Cross-sectional & & Disease \\
& & Exposure & \\
\hline
\end{tabular}

Figure 3 . Overview of the 3 commonly used designs in observational studies.

experimental studies the events are influenced and the effect of the interventions of subjects are investigated. Experimental studies include clinical trials (Pocock 1983), field trials and laboratory studies. Experimental studies are usually performed in order to compare the effect of different treatments on the outcome and stronger conclusions/inferences can usually be drawn compared to observational studies. Under experimental conditions problems with confounders can be reduced or eliminated by careful selection of subjects (e.g. same breed, gender and age). Observational studies can be either prospective (data are collected forward in time) or retrospective (data refer to past events) whereas experimental studies are always prospective.

The 3 basic designs used in observational studies are cohort, case-control and cross-sectional designs, as illustrated in Fig. 3. In a cohort study, groups of subjects with different levels of the study factor (exposure) are included and information on the development of the outcome e.g. of a particular disease (or condition) for a given period of time is collected prospectively. In a case-control study a number of subjects with the disease (cases) are identified along with unaffected subjects (controls). Hereby, cases and controls are selected from 2 different populations. Study factors are collected retrospectively. In cross-sectional studies subjects are selected from the population without know- ledge on the study factors or disease status. The current disease status and (present or past) exposure level are collected at the same time. Comparison of the basic designs and other designs used in observational studies, and a discussion of advantages and disadvantages of the different designs are discussed by e.g. Kleinbaum et al. (1982), Woodward (1999) and Altman (1991).

The simplest intervention design (experimental study) is a parallel group design, where subjects are allocated to one of 2 (or more) treatments. All subjects within a treatment group receive the same treatment. Otherwise, all subjects are treated similarly. If further study factors are to be evaluated, a factorial design can be used. In a factorial design subjects are allocated to the combinations of the factors. Two or more levels for each factor can be used. A parallel design is a one-way factorial design. A Latin square design is a special case of a three-way factorial design having the same number of levels for all 3 factors. An advantage of using a Latin square design is that the number of subjects to be included is limited. However, interactions between the 3 factors cannot be estimated. An alternative to the parallel design is a cross-over design (Senn 1993) in which each treatment is given at different times to each subject. This has the advantage of eliminating the inter-subject variability under certain assumptions, the most important being that of no carry-over effect. 
Table 1. A Latin square design for Example 1: Healing of wounds in horses. Horses are allocated into 4 treatment groups (A, B, C, D) that are combinations of 2 different antibiotics and 2 types of bandage.

\begin{tabular}{lllll}
\hline \multirow{2}{*}{ Leg position } & \multicolumn{4}{c}{ Horse ID } \\
\cline { 2 - 5 } & I & II & III & IV \\
\hline 1 Right fore leg & A & B & D & C \\
2 Left fore leg & B & C & A & D \\
3 Right hind leg & D & A & C & B \\
4 Left hind leg & C & D & B & A \\
\hline
\end{tabular}

In Example 1 (Healing of wounds in horses) different designs can be suggested for the hypothetical experimental study. However, not all designs might be appropriate from a veterinary point of view. Due to practical issues only a limited number of horses can be used, that is less than 10 horses. A Latin square design can be used in which 8 horses are included in 2 groups of 4 horses. An example is given in Table 1.
In Example 2 (Moderate coliform mastitis) 13 cows were included. The design is given in Fig. 4. The study is an observational study with a cohort design, where cows with mastitis were followed for 21 days and evaluated for clinical recovery. Antibiotic treatment is the exposure and clinical recovery after 21 days is the outcome.

In Example 3 (Nematodes in sows and piglets) 39 sows were given different infection levels of nematodes and half of the piglets in each litter were given an infection as well. The structure of the study is given in Fig. 5. The study is an experimental study using a factorial design with 2 factors (one factor with 3 levels for sows, one factor with 2 levels for piglets). Note, that it is not a standard two-way factorial design since piglets are nested within sows, which again are nested within infection levels for sows.

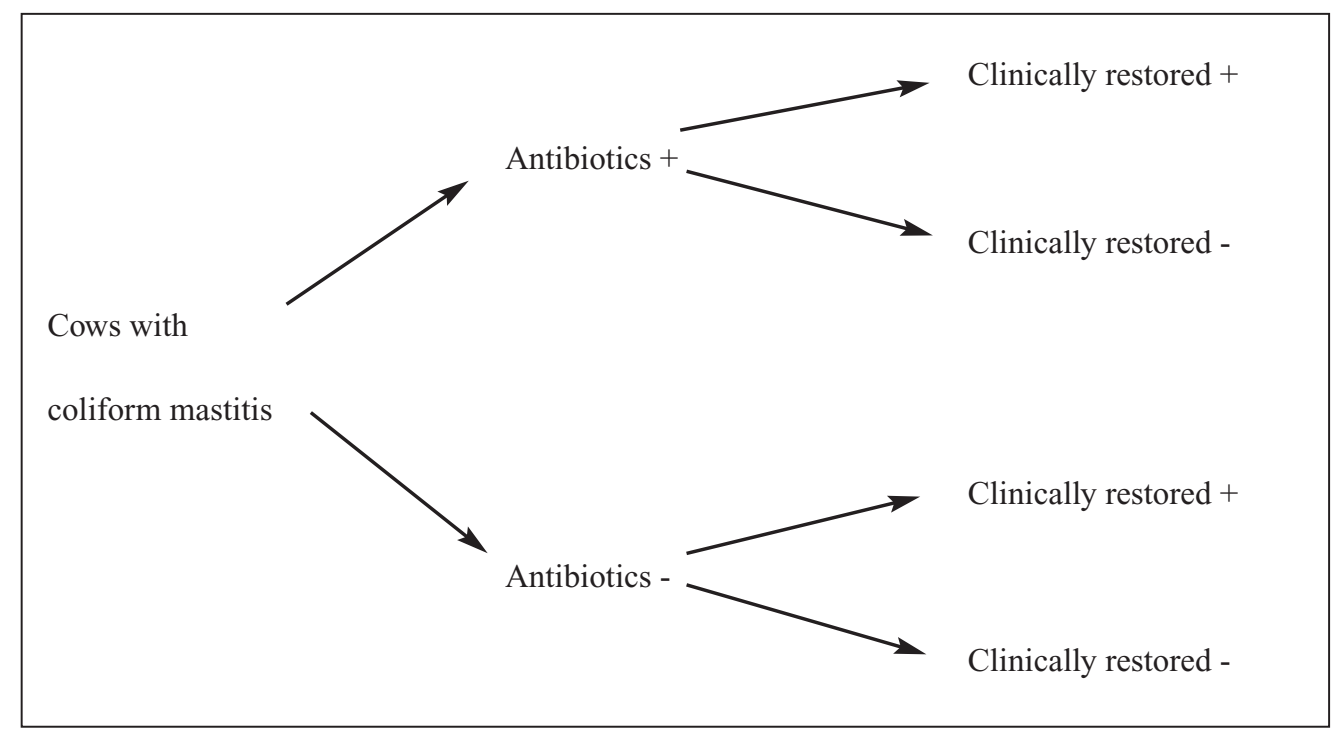

Figure 4. The structure of Example 2: 'Moderate coliform mastitis'. This is an observational study with a cohort design. Antibiotics used is exposure and clinically restored is the outcome. 


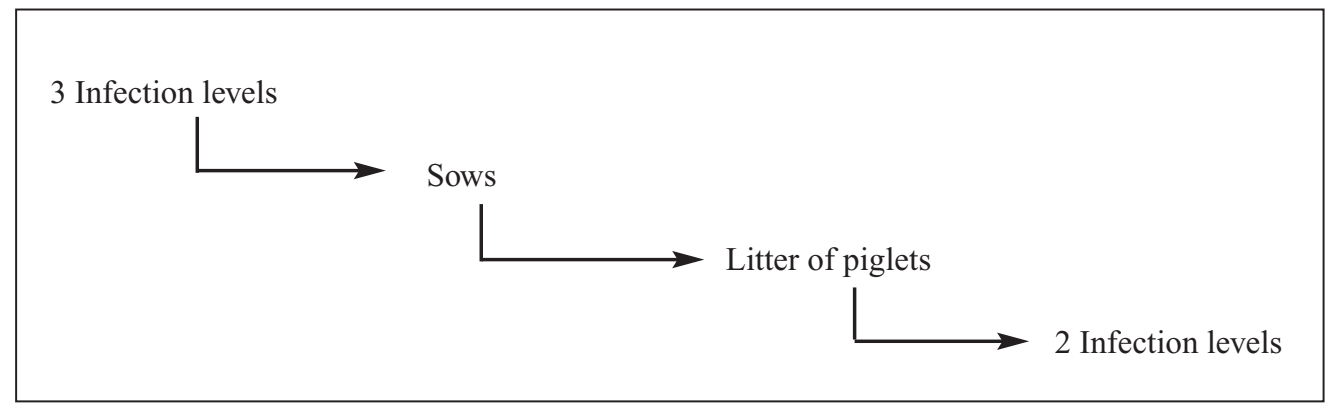

Figure 5. The design for Example 3: 'Nematodes in sows and piglets'. This is an experimental study using a two-way factorial design (one factor with 3 infection levels for the sows, one factor with 2 infection levels for the piglets). Furthermore, the structure is nested (sows are nested within treatments, and piglets are nested within sows).

\section{Sample size and power}

Sample size and power calculations are important in both experimental and observational studies. Whenever a study is being planned there is always a question of sample size, that is how many subjects should be included in order to detect a significant difference of the study factor or treatments. For simple cases, standard equations are available and can be used (e.g. Noordhuizen et al. (1997), Pocock (1983)). Such cases are e.g. comparison of two prevalences or comparison of two means. Calculation of sample size is based on assumptions regarding expected treatment effects, e.g. the expected difference between two means or the expected difference between two prevalences. If no prior knowledge is available from previous studies or publications, expectations or qualified guesses have to be used. Further, the significance level and power, or alternatively the width of the confidence interval, have to be decided as they are used in the sample size calculations as well. The pre-decided significance level is the probability that the null hypothesis is rejected, when it actually is true; Or in more common terms: the probability that a given difference - if not present - would be detected. The power is the probability that the null hypothesis is rejected when it actually is false; In more common terms: the probability that a difference - if present - would be detected. A significance level of $5 \%$ and power of $80-90 \%$ are commonly used in the sample size calculations. An alternative to sample size calculations is calculation of power for varying sample size and/or expected effects (Woodward 1999). In some cases exact equations can be used. However, power calculations can also be performed using simulation, when no standard equations are readily available. In the case of simulation the power is not found exactly but is found as an estimate. If the number of simulations is sufficiently high, the exact and simulated results are very similar. As a rule of thumb 100 simulations will give a power estimate with $95 \%$ confidence limits of \pm 10 percentage-points, while 1000 simulations will give $95 \%$ confidence limits of \pm 3 percentage-points.

In many studies collection of data is repeated in time for each subject in order to evaluate the long-term effects. However, it is important to note that an increasing number of repeated measures does not increase power or efficiency of the test as an increasing number of subjects does. Analogously, in studies which have a nested structure (e.g. herds and animals within 

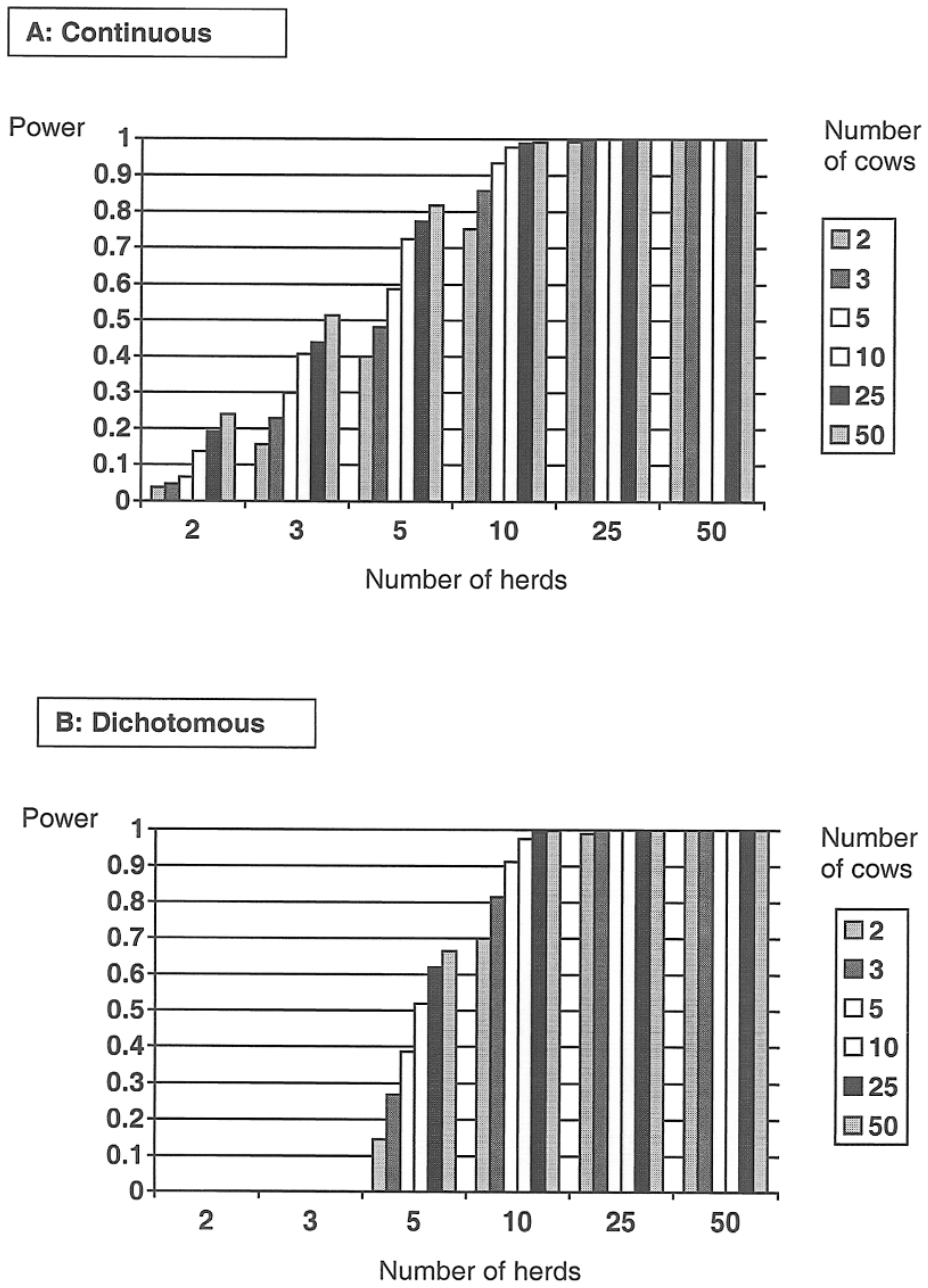

Figure 6. Power for Example 4: 'Milk yield in dairy cows' given for number of herds and number of cows within herds for each breed. A continuous (A) and a dichotomised ( $>18 \mathrm{~kg}$ or $\leq 18 \mathrm{~kg}$ ) outcome (B) have been used.

herds, where treatment regime is given at the herd level) it is often more advantageous from a statistical point of view to include more herds and fewer animals in each herd rather than fewer herds and many animals within each herd.

In Example 4 (Milk yield in dairy cows) it was of interest to calculate the necessary number of herds and number of cows within each herd in order to demonstrate a significant difference in milk yield between the 2 breeds Jersey and Holstein. There is no easily accessible standard equation for this sample size calculation. Instead, simulation of power has been performed 
for a varying number of herds and a varying number of cows within herds. The calculated sample size is for each breed. A continuous outcome (milk yield) and dichotomized outcome (milk yield $>18 \mathrm{~kg}$ or $\leq 18 \mathrm{~kg}$ ) have been used for power simulations. The SAS program used is given in the Appendices 1 and 2. Fig. 6 . shows in both cases that the effect of including many herds with fewer animals is preferable to including fewer herds and many animals within each herd. For the example with 5 herds and 10 animals per herd for each breed the power is $72 \%$ for the continuous outcome and $52 \%$ for the dichotomous outcome. Changing that to 10 herds and 5 animals per herd for each breed the power rises to $93 \%$ for the continuous and $91 \%$ for the dichotomous cases, respectively. The reason for this perhaps surprising result is that the p-value for a breed (or treatment) effect depends heavily on the variance between herds, even though it may be smaller than the variance between animals within herds.

\section{Compliance}

For different reasons a part of the subjects included in the study might drop out before the end of the study (e.g. trading, death). These withdrawals might be a problem in relation to demonstration of significant effects, if the significance is based on a sample size, which has been reduced due to drop out. Therefore, the estimated sample size should be increased in accordance with the expected percentage of withdrawal. If for instance the sample size needed is estimated at 100 subjects, and the expected withdrawal is $20 \%$, in total 125 subjects should be included in the study.

\section{Randomisation}

Randomisation is important (1) to prevent (or reduce) bias (systematic error) and (2) to provide a basis for statistical analysis such as significance tests. In epidemiological research bias is defined as: confounding, misclassification, information bias, and selection bias (see e.g. Kleinbaum et al. 1982, Noordhuizen et al. 1997). Bias can occur in a study if there is preferential assignment of subjects to the study factor(s) in experimental studies or differential selection of subjects in observational studies. Randomisation will ensure that the variation in data is evenly distributed between the subjects. Further, randomisation will help balance the distribution of other variables e.g. confounders such as age, gender and breed. The types of randomisations commonly used are (1) simple (complete), (2) restricted (block), (3) multistage, and (4) stratified. Simple randomisation means that each subject is included without attention to possible confounders. Block randomisation ensures a similar number of subjects within each level of the study factor. Multistage sampling is used when data have a multilevel structure such as herds and subjects within herds. First, herds are randomly selected; secondly, subjects within herds are randomly selected. Stratified randomisation is used when one or more factors are known to have an influence on the outcome, such as age, gender, and breed. The inclusion of subjects is balanced regarding the factors. Stratification might be a problem in studies with small data sets as the number of strata (combinations of the factors used in stratification) can approach or even exceed the number of subjects. An acceptable alternative is minimisation, which is a non-random allocation of subjects to study factors (Altman 1991). Minimisation has advantages over both simple and stratified randomisation, when sample size is small. The factor groups are very similar even for several study factors.

The study in example 1 (Healing of wounds in horses) used a latin square design. There are a number of possible latin squares with 4 levels of each factor. First, one of these latin squares is randomly selected (simple random selec- 
tion). Next, the 4 horses are block randomised to the combinations of the 2 remaining factors. In example 2 (Moderate coliform mastitis) simple randomisation was used to allocate cows to antibiotic treatment or not. Simple randomisation was also used in example 3 (Nematodes in sows and piglets) in order to allocate sows to the 3 infection levels and piglets to the 2 infection levels. Example 4 (Milk yield in dairy cows) used a two-stage sampling. Herds were randomly selected among all Danish dairy herds. Cows within herds were randomly selected.

\section{Data quality}

Data quality is always very important, even for a large data set. However, in most studies the importance of data management is underestimated. Inappropriate data quality may lead to statistical analysis based on incomplete and erroneous data resulting in wrong conclusions. The importance of efficient data management can therefore not be stressed enough. Data management includes collection of data, organising data in a database and data control (checking data). When constructing the database, common ways to minimize the number of typing errors are by (1) entering data twice and identify discrepancies or (2) performing a complete proofreading of data entered into the database. In the following data control and correction of data will be discussed. Problems with clinical examinations will be discussed in relation to small data sets. Data management including data control has been a very important part (time and cost) of human clinical trials (see e.g. Pocock (1983) and Altman (1991)). In veterinary medicine not much has been written about data management and how to perform data control. However, e.g. Rothman \& Greenland (1998) give some suggestions.

Data quality is especially important when dealing with small studies. Identification of strange observations is nearly impossible, due to the limited number of observations. For a large data set it is often possible to identify a strange observation by evaluating the distribution of data and identify a strange observation as being outstanding from the remaining observations. However, with a small number of subjects, it is often difficult to evaluate if one observation is dubious by comparing it to the (empirical) distribution of the remaining observations.

\section{Data control}

Data control will usually result in improved data quality. Data control can be performed during data collection as well as after data collection. Performing data control during collection of data might identify specific problems with some of the recordings which can be changed and thereby improve the validity and quality of future recordings. Data control should always be performed after data collection and before statistical analysis is initiated. Depending on the type of variables different procedures of data checking can be used. The types of variables can be divided into qualitative (dichotomous, nominal, ordinal), quantitative (discrete and continuous) and miscellaneous (e.g. dates). Qualitative data as breed and gender have pre-specified values or codes. Therefore, qualitative data can be checked by identifying impossible values. For quantitative data as weight and milk yield it is not possible to precisely identify incorrect values. It should, however, be possible to specify a range with lower and upper limits of reasonable values for the variable. However, values outside the range are not necessarily incorrect, they are just flagged for possible further examination. Data control also includes evaluating the frequency distribution and completeness of data. Consistent ordering of dates can be checked. Logical control can be used in order to evaluate consistency of the data set (e.g. parity given for cows only and not for bulls) and identifying strange 
Table 2. The influence of data quality on the association between antibiotics used and clinically restored on day 21 in Example 2: Moderate coliform mastitis. One of the clinical evaluations has been changed and the influence on the significance level ( $\mathrm{p}$-value) is given. Table A is the original data, tables B-D are modifications. Figures in boldface and italic are clinical examinations that have been changed.

\begin{tabular}{lccc}
\hline & & \multicolumn{2}{l}{ Clinically restored } \\
\cline { 3 - 4 } & & Yes & No \\
\hline Anti- & Yes & 7 & 0 \\
biotic & No & 2 & 4 \\
\hline
\end{tabular}

Test for association : $\mathrm{p}=0.020$.

\begin{tabular}{lclc} 
& & \multicolumn{2}{c}{ Clinically restored } \\
\cline { 3 - 4 } & & Yes & No \\
\hline Anti- & Yes & 7 & 0 \\
biotic & No & $\mathbf{1}$ & $\mathbf{5}$ \\
\hline
\end{tabular}

Test for association: $\mathrm{p}=0.005$

observations (e.g. scatterplots of the relation between 2 continuous variables and time profiles for each subject).

\section{Correction of data}

Whenever a wrong or strange observation has been identified a decision has to be made regarding data editing. Suspicious values should be checked with the original data forms (if they exist) and errors corrected. Other values might be left unchanged or coded as missing information. Elimination of subjects and/or values should in general be avoided.

\section{Clinical examinations}

Clinical examinations made by e.g. the veterinary practitioner are to some extent subjective and might be wrong (misclassification bias). Technically speaking, each veterinary practitioner might have his/her own sensitivity (ability to assess true positive) and specificity (ability to assess true negative). In small data sets it might have a large influence if some of the clinical examinations are wrong. In order to improve the clinical examinations, the agreement

\begin{tabular}{lccc}
\hline \multirow{2}{*}{ B } & \multicolumn{2}{l}{ Clinically restored } \\
\cline { 3 - 4 } & & Yes & No \\
\hline Anti- & Yes & $\mathbf{6}$ & $\mathbf{1}$ \\
biotic & No & 2 & 4 \\
\hline
\end{tabular}

Test for association: $\mathrm{p}=0.100$

\begin{tabular}{lccc} 
& & \multicolumn{2}{c}{ Clinically restored } \\
\cline { 3 - 4 } & & Yes & No \\
\hline Anti- & Yes & 7 & 0 \\
biotic & No & $\mathbf{3}$ & $\mathbf{3}$ \\
\hline
\end{tabular}

Test for association: $\mathrm{p}=0.070$

(intra- and inter-observer variations) between e.g. two observers can be calculated. Similarly, agreement between repeated evaluations performed by the same observer at different times can be evaluated.

\section{Statistical analysis}

The main aim of a statistical analysis is to use the information from a (random) sample of subjects to make inferences about the relevant population. Most analyses will include hypothesis testing and estimation of the effect of the study factors (e.g. treatments). Having a large data set often implies robust analyses and results, meaning that we can obtain the same results in different analyses. In case of a small data set even small changes in the analysis and/or changes in the data set might inflate the results. Testing the effect of a study factor (e.g. two treatments) is done by comparison of the difference between the treatments relative to the standard error of the difference. Sample size and standard error are inversely related implying a large standard error when sample size is small. A small study may therefore fail to detect a (significant) dif- 
ference that is really present. With a decreasing sample size we will usually see an increasing p-value towards non-significance. In the following the choice of method, improvement of analysis and non-parametric tests will be discussed.

The importance of quality of data is illustrated by Example 2: Moderate coliform Mastitis (Table 2). The association between using antibiotics and being clinically restored on day 21 was significant $(\mathrm{p}=0.02$ using Fisher's exact test). If just one of the clinical evaluations is changed from restored to not restored or visa versa, the association might not be significant any longer. In 2 of 3 cases where one evaluation is changed, a non-significant association is seen.

\section{Choice of method}

The choice of method depends on the type of outcome (also called the response variable). A dichotomous outcome is a qualitative variable with only two levels such as diseased yes/no. A continuous outcome is a variable, which can take all possible values such as weight gain and milk yield. However, the continuous outcome often has a lower and/or upper bound (e.g. milk yield cannot be negative). In case of a dichotomous outcome the relevant analyses include $\chi^{2}$ test, Fisher's exact test, McNemar's test and logistic regression. The relevant analyses with a continuous outcome include the t-test (for paired and un-paired observations), analysis of variance and linear regression. With other types of outcomes such as ordinal or nominal variables with more than two levels ordinal logistic regression, multinomial logistic regression and loglinear models can be used (e.g. Hosmer \& Lemeshow 2000, Agresti 1990).

\section{Improvement of analysis}

Correct model specification is crucial in all analyses. The study factors and possible con- founders should be included in the model. However, in a study with a small number of subjects, it might be impossible to include confounders as well as study factors in the same analysis. The number of variables, which can be included in the model, depends on the number of subjects. Depending on the number of levels for each factor, 2-3 study factors can be evaluated with e.g. 20 subjects. Confounding might be difficult to deal with having small data sets. Possible confounders are often evaluated by including these variables in the analysis or by performing analyses stratified by these variables. However, having fewer subjects it might be impossible to include confounders due to limited degrees of freedom. Repeated measures are often recorded in order to evaluate long term effects. However, many repeated measures can generally not compensate for a limited number of subjects.

Logistic regression analysis of a dichotomous outcome can be improved using exact logistic regression (LogXact).

In a study including herds and subjects within herds it can be impossible to include herds as a random effect if the number of subjects is limited.

If strange observations have been identified in the data control and no obvious explanation can be found, the importance of the strange observation can be evaluated by analysis. In the analysis the strange observation can be included or left out in turn. Differences between the 2 analyses can help identify an influential observation. For small data sets, however, elimination of even a "normal observation" might cause dramatic differences in the results. This approach is therefore not possible for small data sets. Consistent evaluation of the influence of strange observations is therefore nearly impossible for small data sets. This indicates further the importance of having high quality data. Model validation can be used in order to vali- 
date the results. Ideally, this is done using the original subjects for estimation of the model. Validation is then performed using new subjects, by estimation of the outcome for the new subjects using the developed resulting model. Differences between the estimated and observed outcome for the new subjects indicate the performance of the resulting model. However, it is often not feasible to collect validation data and the original data must be used for the whole validation process. With a large data set, this will normally be done by dividing data into 2 subsets, one subset is used for developing and estimating the model (learning subset), the other subset is used to validate the model (test subset). The difference between the observed and predicted values for the test subset is calculated and is used as an indicator of model quality. The learning subset often comprises $1 / 2$ or $2 / 3$ of the complete data set depending on number of observations in the complete data set. For a small data set, this is often not possible, as the number of observations in the learning subset may be too small to estimate the model. Instead, validation can be performed using cross-validation (Weisberg 1985). Here, one of the most commonly used methods is the so-called leaveone-out technique.

Simulation and bootstrapping are further methods that can be used in order to understand, improve, and validate the models.

\section{Non-parametric methods}

Non-parametric methods are also called distribution-free tests and rank methods. For the simplest parametric tests there are corresponding non-parametric tests e.g. Mann-Whitney's test and Wilcoxon's test correspond to t-test for unpaired observations, Wilcoxon's signed rank test corresponds to t-test for paired observations, Kruskall Wallis's test and Friedman's test correspond to one-way and two-way analysis of variance, respectively. A non-parametric test is a test where no assumptions regarding the outcome have to be fulfilled. However, there are still assumptions which must be fulfilled (Conover 1980). In general, the non-parametric tests are not as informative as parametric methods because the non-parametric methods use ranks instead of original values. The non-parametric methods are therefore mainly used for testing hypotheses and not for estimation.

If the assumptions for performing a parametric test are satisfied, the non-parametric tests are not as efficient as the parametric. The relative efficiency between 2 similar tests can be calculated as the sample size needed using one test compared to the sample size needed for the second test under similar conditions (Conover 1980). The relative efficiency of non-parametric tests compared to the corresponding parametric test is often small. Therefore, if the assumptions for performing a parametric test are fulfilled, it is easier to detect significant effects using the parametric test compared to the relevant non-parametric methods. Furthermore, parametric methods offer a much richer class of models than non-parametric methods.

\section{Acknowledgements}

The authors would like to thank SM Thamsborg and PH Andersen from The Royal Veterinary and Agricultural University, Denmark and J Katholm, Vivild, Denmark for providing us with examples of small data sets. We would also like to thank an anonymous reviewer for numerous comments which helped clarify the paper.

\section{References}

Agresti A: Categorical data analysis. John Wiley \& Sons, New York 1990.

Altman DG: Practical statistics for medical research. Chapman \& Hall, London 1991.

Conover WJ: Practical non-parametric statistics, 2.ed. John Wiley \& Sons, New York 1980.

Hosmer DW, Lemeshow S: Applied logistic regression, 2.ed., John Wiley \& Sons, New York, 2000.

Katholm J, Andersen PH: (2001) Treatment of coliform mastitis in bovine practice - can antibiotics 
be avoided? In: Proceedings from The 11th International Conference on Production Diseases in Farm Animals, 2001, 12-16 August, Frederiksberg, Denmark. Acta vet. scand. 2003, Suppl. 98.

Kleinbaum DG, Kupper LL, Morgenstern H: Epidemiologic research. Van Nostrand Reinhold, New York 1982.

Noordhuizen JPTM, Frankena K, van der Hoofd CM, Graat EAM: Application of quantitative methods in veterinary epidemiology. Wageningen Press, Wageningen 1997.

Pocock SJ: Clinical trials. A practical approach. John Wiley \& Sons, New York 1983.

Senn S: Cross-over trials in clinical research. John Wiley \& Sons, New York 1993.

Thamsborg SM, Mejer H, Roepstorff A, Ersbøll AK, Eriksen L: Effects of nematodes on health and productivity of outdoor sows and suckling piglets. In: Proceedings from the 18th International Conference of the WAAVP, p53, 2001. Held in Stresa, Italy.

Weisberg S: Applied linear regression. Wiley \& Sons, New York 1985.

Woodward M: Epidemiology. Study design and data analysis. Chapman \& Hall/CRC, London 1999.

Rothman KJ, Greenland S: Modern Epidemiology. Lippincott - Raven Publishers, Philadelphia 1998.

\section{Sammendrag \\ Epidemiologiske studier baseret på små datascet - en statistikers synspunkt.}

Vi betragter tre vigtige trin $i$ et studie, som har relevans for den statistiske analyse. De er: design af studiet, data kvalitet og statistisk analyse. Mens statistisk analyse ofte bliver betragtet som et vigtigt element i litteraturen og valget af statistisk metode får megen opmærksomhed, så synes der at blive lagt mindre vægt på design af studiet og nødvendig stikprøvestørrelse. Endelig bliver et meget vigtigt element, nemlig undersøgelse og validering af de indsamlede data's kvalitet, oftest overset.

Eksempler fra veterinær epidemiologi og anbefalinger for hvert af trinnene bliver givet sammen med relevante referencer til litteraturen.

Peer reviewed contribution to 11. International Conference on Production Diseases in Farm Animals, 12-16 August 2001, The Royal Veterinary and Agricultural University, Frederiksberg, Denmark.

Reprints may be obtained from: Annette Kjær Ersbøll, Department of Animal Science and Animal Health, The Royal Veterinary and Agricultural University, Grønnegårdsvej 8, DK-1870 Frederiksberg C

Denmark. E-mail: ake@kvl.dk, tel: +45 352830 21, fax: +45 35283022. 
Appendix 1: Nested example using continuous data. Program written in SAS for calculation of sample size.

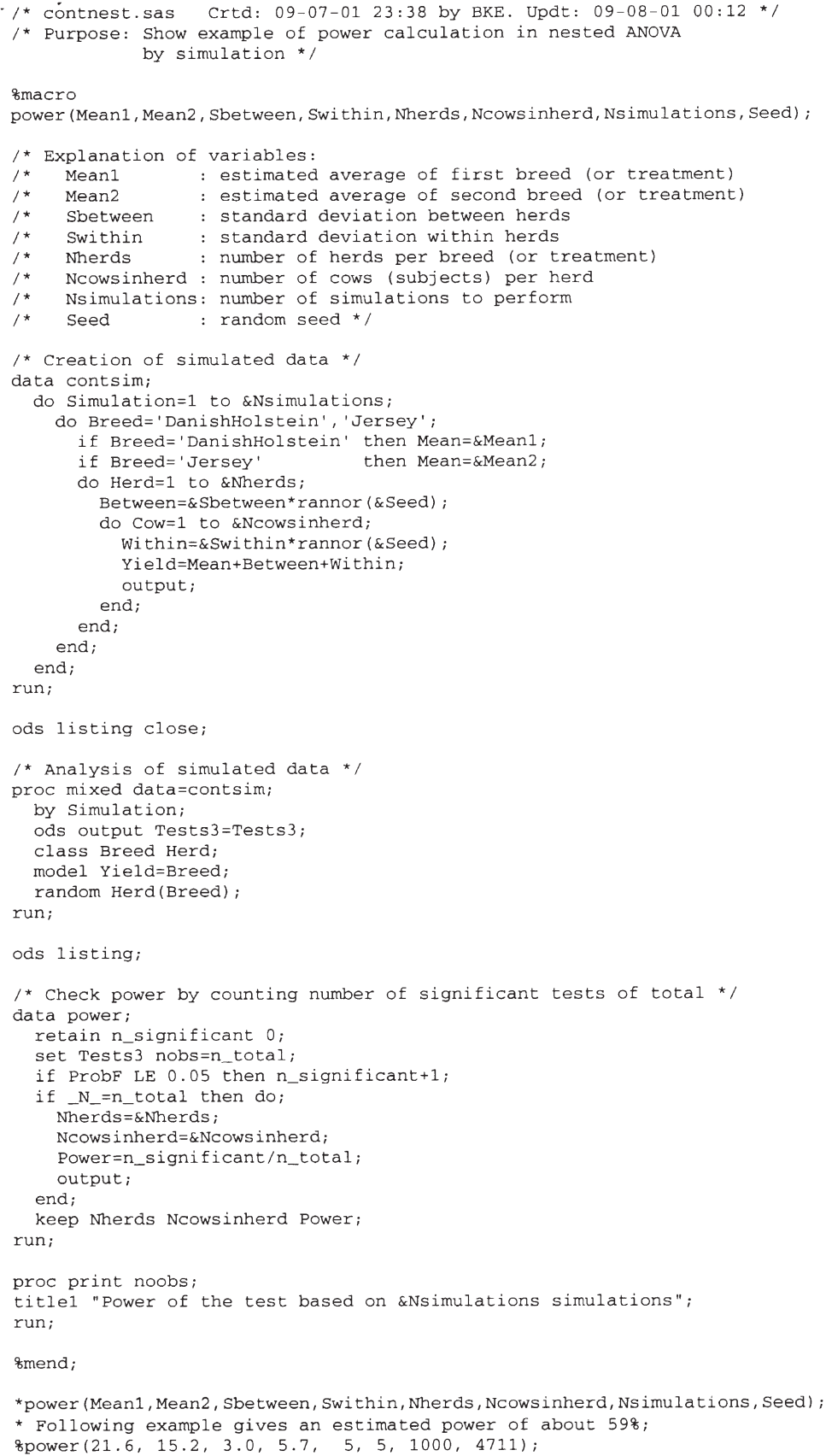


Appendix 2: Nested example using dichotomous data. Program written in SAS for calculation of sample size.

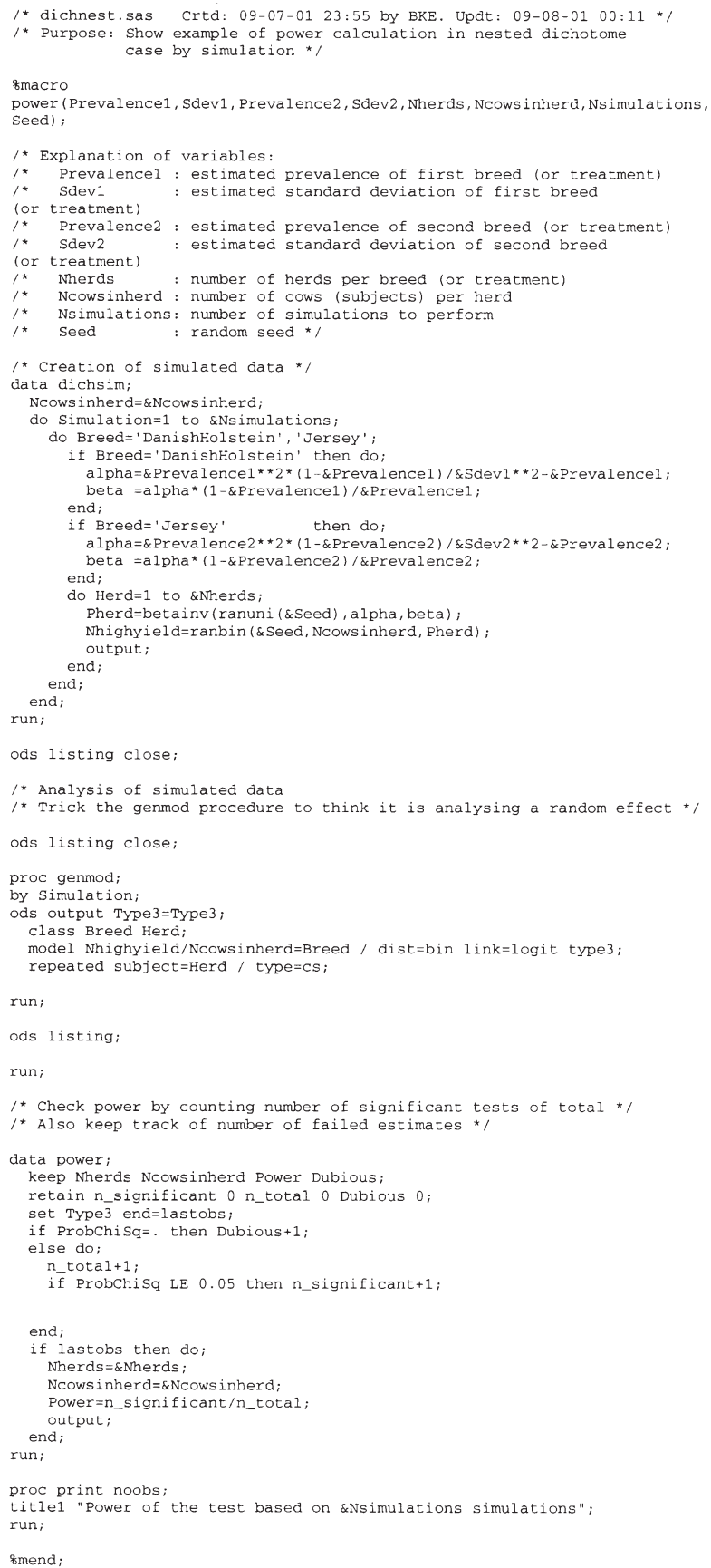

